Vol. 35 (1), 2017 (ISSN 0254-9247)

\title{
Participación política: el aporte discriminante de actitudes ideológicas, valores y variables sociopsicológicas
}

\author{
Patricia Sorribas ${ }^{1}$, Silvina Brussino ${ }^{2}$ \\ Universidad Nacional de Córdoba-Argentina
}

El presente trabajo identifica variables que discriminan entre las personas que participan y quienes no participan políticamente, considerando dimensiones electorales y no electorales de la participación política en Córdoba, Argentina. Se realizó un estudio por encuesta en una muestra representativa de la población $(\mathrm{N}=450)$. El análisis discriminante permitió reconocer el aporte del autoritarismo de derecha (RWA), colectivismo, colectivismo político, interés político y eficacia política participativa, a dicha diferenciación en dos dimensiones de la participación: electoral-de campaña y de contacto con organizaciones y referentes políticos. Estos resultados evidencian la relación del RWA con otros comportamientos políticos diferenciados de las preferencias partidarias y comprueban la relación de diferentes valores y de una medida participativa de la eficacia política con amplios repertorios participativos. Palabras clave: participación política, autoritarismo de derecha, orientación a la dominancia social, colectivismo e individualismo, eficacia política participativa.

Political Participation: discriminant contribution of ideological attitudes, values and socio-psychological variables

This paper identifies variables that discriminate between participants and non-participants considering electoral and non-electoral dimensions of political participation in Córdoba, Argentina. A study was conducted by survey on a representative sample of the population ( $\mathrm{N}$ = 450). The discriminant analysis allowed recognizing the contribution of right-wing authoritarianism (RWA), collectivism, political collectivism, political interest and of participatory measure of "political efficacy", to such differentiation in two dimensions of participation: electoral-campaign and contact with organizations and political figures. These results show the relationship of RWA with different political behavior of party preferences; and verify the

1 Doctora en Psicología e investigadora asistente CONICET en Equipo de Psicología Política del Centro de Investigaciones de la Facultad de Psicología de la Universidad Nacional de Córdoba (CIPSI). Dirección postal: Enfermera Gordillo y Enrique Barros, Ciudad Universitaria, CP 5000, Córdoba, Argentina. Contacto: patricia.sorribas@conicet.gov.ar

2 Doctora en Psicología e investigadora independiente CONICET en Equipo de Psicología Política del Centro de Investigaciones de la Facultad de Psicología de la Universidad Nacional de Córdoba (CIPSI). Dirección postal: Enfermera Gordillo y Enrique Barros, Ciudad Universitaria, CP 5000, Córdoba, Argentina. Contacto: brussino9@gmail.com 
relationship of different values and a participatory measure of "political efficacy" with broad participation repertoires.

Keywords: political participation, right-wing authoritarianism, social dominance orientation, collectivism and individualism, political efficacy.

\section{Participaçáo política: a contribuiçáo discriminante de atitudes ideológicas, valores e variáveis sócio-psicológicos}

Este documento identifica as variáveis que discriminam entre os participantes e não participantes, considerando dimensóes eleitorais e náo-eleitorais de participaçáo política em Córdoba, Argentina. Um estudo foi realizado por levantamento de uma amostra representativa da população $(\mathrm{N}=450)$. A análise discriminante permitiu reconhecer a contribuição de "autoritarismo de direita" (RWA), colectivismo, "coletivismo político"; política e interesse "política participativa eficácia”. Essas variáveis discriminar participantes em duas dimensôes de participação: eleitorais - "campanha” e contato com organizaçôes e figuras políticas. Estes resultados mostram a relação de RWA com outro comportamento político diferenciado das preferências partidárias e verificar a relação de valores diferentes e uma medida de eficácia política participativa, com amplos repertórios de participação.

Palavras-chave: participação política, autoritarismo de direita; orientação para a dominância social, coletivismo eo individualismo, eficácia política participativa. 
La producción en torno a la participación política (PP) permite reconocer que su conceptualización contempla desde definiciones sumamente restrictivas (Verba \& Nie, 1972) que implican un repertorio acotado a la participación electoral y partidaria con énfasis en el voto (Krampen, 2000; Pearl \& Anderson, 2007), hasta definiciones mucho más amplias que integran variados repertorios participativos (Cohen, Vigoda \& Samorly, 2001; Milner, 2010; Norris, 2002). A su vez, las concepciones que contemplan repertorios ampliados incluyen prácticas de influencia sobre los responsables políticos a través de modos más directos y una variedad de ejercicios de autoorganización que se distinguen por estar orientados hacia el bien colectivo y porque a menudo producen beneficios a aquellos que los utilizan, de allí que también se los califique como participación cívica (John, Fieldhouse \& Liu, 2011).

La conceptualización de la PP es relevante pues delimita la amplitud que alcanza su repertorio y dentro de este las dimensiones en las que puede diferenciarse. $\mathrm{Al}$ respecto cabe aclarar que en el presente estudio no se adoptan las "más comunes de las clasificaciones" de esas dimensiones (Van der Meer \& Van Ingen, 2009, p. 286) es decir aquellas que diferencian la PP en convencional y no convencional (FernándezPrados \& Rojas-Tejada, 2003; Mannarini, Legittimo \& Talò, 2008; Robnett, 2007) o en institucional y no institucional (Bolzendahl \& Coffé, 2010; Marien, Hooghe \& Quintelier, 2010). Contrariamente, se asume el carácter multidimensional de la PP, en coincidencia con una vasta producción en este campo de estudios.

\section{La multidimensionalidad de la participación política}

La multidimensionalidad de la PP constituye un hallazgo ampliamente compartido en la literatura especializada (Claggett \& Pollock, 2006; Gallego, 2007; Morales, 2005; Sabucedo \& Arce, 1991; Torcal, 
Montero \& Teorell, 2006; Van der Meer, Van Deth \& Scheepers, 2009). Inclusive, se ha recurrido a la clasificación convencional y no convencional para identificar cuatro dimensiones, dos para cada una de esas categorías (ver Sommuano Ventura, 2005). Particularmente en Argentina se han desarrollado estudios conducentes a la identificación de la multidimensionalidad de la PP (Brussino, Rabbia \& Sorribas, 2008; Brussino, Sorribas, Rabbia \& Imhoff, 2013; Delfino, Zubieta \& Muratori, 2013). En la ciudad de Córdoba, Argentina, en un estudio reciente (Sorribas, 2014) se identificaron, mediante un análisis factorial de componentes principales, cuatro dimensiones de la PP, que logró explicar un $46 \%$ de la varianza. La primera correspondió a acciones realizadas dentro de partidos políticos y orientadas a campańas electorales (por ej.: distribuir propaganda política) que han sido ampliamente reportadas en la literatura relativa a campańas (Sabucedo, 1996; McAtee \& Wolak, 2011; Van der Meer et al., 2009), al activismo partidario (Whiteley \& Seyd, 2002) y a la membrecía partidaria (Letki, 2002; Morales, 2005; Torcal et al., 2006). La segunda incluyó acciones de contacto con organizaciones y referentes políticos con diferentes niveles de formalización (por ej.: contacto con Centros Vecinales, hacer llegar ideas a concejales, intendentes, legisladores o funcionarios) similares a los de otros estudios (Dalton, 2006 y 2008; Marien et al., 2010; Morales, 2009; Torcal et al., 2006; Van der Meer et al., 2009). La tercera integra comportamientos contenciosos y reivindicativos relativos al mundo del trabajo y asociados con organizaciones sindicales (por ej.: cortes de calles, paros-huelgas y contactar a gremios o sindicatos). Esta dimensión puede asimilarse al repertorio de protesta (Brussino et al., 2013; Gallego, 2007; Morales, 2005; Torcal et al., 2006; Van der Meer et al., 2009), a la participación activista (Velásquez, Martínez \& Cumsille, 2004) o a acciones no institucionalizadas (Bolzendahl \& Coffé, 2010). A su vez, repertorios similares fueron establecidos por Sabucedo y Arce (1991) y Zmerli (2002). La cuarta dimensión contempla modalidades de participación orientadas a ejercer influencia - mediante diferentes acciones — sobre las decisiones del Poder Legislativo (Nacional o Provincial) y constituye un ejemplo claro de un 
repertorio más coyuntural. Mayoritariamente refiere a acciones que, si bien intentan impactar sobre el sistema representativo, lo hacen desde afuera del sistema (Van der Meer \& van Ingen, 2009), ya que en ningún caso se realizaron acciones más orgánicas dentro de partidos políticos. Por tratarse del antecedente más reciente a nivel local, en el presente trabajo se considera esta dimensionalización.

\section{Factores que diferencian entre participantes y no participantes}

La literatura relativa a factores explicativos de la PP es sumamente cuantiosa. De todos modos, el aporte de algunos constructos está fuera de discusión. Tal es el caso de variables "sociopsicológicas" — según la denominación de John, Fieldhouse y Liu (2011) — como la eficacia política (Klesner, 2007; Krampen, 2000; Mannarini et al., 2008); interés político (Brussino, Rabbia \& Sorribas, 2008; Gabriel \& Van Deth, 1998; Marien et al., 2010; Rodríguez, Sabucedo \& Costa, 1993; Sabucedo, 1996; Vráblíková, 2010), conocimiento político (Brussino, Medrano, Sorribas \& Rabbia, 2011; Delli Carpini \& Keeter, 1993) y la confianza política (Grossi \& Ovejero, 1994; John et al., 2011; Mannarini et al., 2008; Van der Meer \& van Ingen, 2009; Zmerli, 2002).

La eficacia política es considerada como la creencia subjetiva de un individuo o grupo de poseer capacidades para participar e influir en el curso de los sucesos políticos (Krampen, 2000). Según la revisión de Mannarini et al. (2008, p. 99), diversos estudios desde la década de 1970 hasta mediados de la primera década del siglo XXI reportan que "bajos niveles de autoeficacia desaniman a la gente a asumir un papel activo, es decir que más bien los alienta a retirarse hacia el ámbito privado, tanto a individuos como a miembros de grupos de acción social”. Wollman y Stouder (1991) reportan que los análisis en conjunto de las diferentes investigaciones que desarrollaron, señalan que en 9 de 10 estudios observan que variables como el locus de control resultaban demasiado globales para ser buenos predictores de aspectos específicos del comportamiento político. De allí que estos autores justifiquen el desarrollo de escalas que impliquen mayor especificidad situacional en los sentimientos de eficacia. 
El interés político de acuerdo a Gabriel y van Deth (1998) se trata de una sensación de curiosidad por los asuntos políticos. Existe acuerdo en que uno de los hallazgos mejor establecidos en la investigación sobre la opinión pública es la posición crucial de este constructo en los esquemas que unen los factores sociales y psicológicos con las actitudes políticas y el comportamiento (Rodríguez et al., 1993). A su vez el interés político que posee un sujeto o grupo sobre los asuntos políticos, o al menos, sobre los resultados de estos, ha sido señalado como una variable que predice la PP (Brussino, Rabbia \& Sorribas, 2008; Delfino et al., 2013; Sabucedo, 1996; Soule, 2001; entre otros).

El conocimiento político puede ser definido como el nivel de entendimiento que poseen los ciudadanos sobre el funcionamiento político en el cual se encuentran inmersos. Los ciudadanos se diferencian tanto por sus niveles de conocimiento político como por el contenido de esta información: temas de actualidad, individuos activos en la política y en el gobierno, principios constitucionales que subyacen al gobierno, funcionamiento del sistema político, entre otros. A pesar de esta última diferencia Delli Carpini y Keeter (1993) se inclinan hacia un modelo unidimensional de este constructo, es decir cuando un sujeto conoce sobre un aspecto político es capaz de conocer sobre otros aspectos políticos.

La confianza política se define como la fe que tienen los ciudadanos en las acciones y la gente que representa al gobierno. La confianza en las instituciones gubernamentales enfatiza la percepción de que el sistema político y las autoridades son responsables por los intereses y demandas del público (Reef \& Knoke, 1999).

Otros constructos cuentan con menos antecedentes o han sido estudiados principalmente en relación con la dimensión electoral de la PP (voto, preferencias partidarias, comportamiento de campaña) y en menor medida con dimensiones no electorales. Tal es el caso de los valores colectivismo-individualismo y de las "actitudes ideológicas" — de acuerdo a la denominación provista por Sibley y Duckitt (2009) y Duckitt, Wagner, du Plessis y Birum (2002) - para referirse al autoritarismo de derecha (RWA) y a la orientación a la dominancia social (SDO). 
En cuanto al individualismo y colectivismo (I/C), estos valores pueden ser distinguidos principalmente en relación con las características de horizontalidad o verticalidad. Esta distinción hace foco sobre la extensión en la cual el poder y la (des)igualdad en las relaciones sociales o entre las personas es percibida dentro de las culturas. El aspecto vertical consiste en los valores poder y logro, mientras la horizontalidad contempla los valores benevolencia y universalismo (Paquet \& Kline, 2009). A su vez, en América Latina y de acuerdo a los aportes de Schwartz (2006) se reportan niveles más altos en los valores jerarquía y arraigo, presumiblemente los principales componentes del colectivismo. Junto a este dato se registra un menor nivel en el reporte de la autonomía intelectual, probablemente el principal componente del individualismo.

Por otra parte, las dimensiones horizontalidad y verticalidad del I/C resultan pertinentes ya que pueden interpretarse como creencias acerca de la igualdad/desigualdad entre los miembros de un grupo cultural (Komarraju \& Cokley, 2008). El colectivismo en particular se asocia a actividades legales no institucionalizadas (por ej.: firma de petitorios) y protestas debido a que las "normas sociales colectivas fuertes engendran un sentido de deber hacia los vecinos y la comunidad que puede llegar a faltar en las personalidades más individualistas" (John et al., 2011, p. 249). La relación entre I/C y el RWA ha sido muy documentada (Schimmack, Oishi \& Diener, 2005) y Bourgeois (2002) reporta evidencia de la relación entre I/C y la orientación a la dominancia social (SDO). Por otra parte, los valores I/C y estas actitudes ideológicas han sido asociadas con un comportamiento más convencional como la afiliación partidaria (Bourgeois, 2002).

Estos valores también han sido evaluados específicamente en relación con el dominio de lo político. La escala que evalúa el colectivismo político (Sorribas, 2012) enfatiza el carácter colectivo tanto de las acciones políticas como de sus fines. Es decir, el carácter colectivo no refiere a los límites de grupos exclusivos o alentados por intereses particulares, sino que refiere a intereses generales y bienes públicos pretendidos para la sociedad como principal colectivo. Por su parte, 
el individualismo político acentúa el autointerés o la obtención de beneficios personales como principales motivaciones para la acción política.

Siguiendo a Duckitt et al. (2002) la SDO y el RWA constituyen actitudes ideológicas que expresan las motivaciones derivadas de la competencia grupal basada en la dominancia y la superioridad (SDO) y las motivaciones derivadas de la amenaza para la seguridad colectiva y la cohesión social (RWA). Mavor, Louis y Sibley (2010) reportan una estructura bifactorial de la escala SDO, siendo estas dimensiones las de orientación hacia la igualdad social (OI) y orientación a la dominancia grupal (OD). En relación con el RWA cabe mencionar que a pesar de que la teorización propone la unidimensionalidad del constructo y tres componentes subyacentes, según Mavor et al. (2010) se ha obtenido evidencia mediante análisis factoriales de dos factores que son consistentes con medidas fuertes de agresión y convencionalismo. En tal sentido Walter, Stone y Bourgeois (1996) reportan dos grandes factores, uno formado por los ítems autoritarios y el otro por los ítems democráticos.

Ambas actitudes han sido asociadas con diversos valores políticos (Crowson, Thoma \& Hestevold, 2005; Lehmiller \& Schmitt, 2007; Mavor et al., 2010), con variables cognitivas (Sibley \& Duckitt, 2009), con medidas de personalidad (Heaven \& Bucci, 2001) y con actitudes y posicionamientos políticos-partidarios (Cárdenas, Meza, Lagues \& Yañez, 2010; Crowson et al., 2005) de allí su valor para el presente trabajo. A diferencia de los antecedentes reportados, aquí se intenta comprobar el aporte de estos constructos a la discriminación entre participantes y no participantes de distintas dimensiones participativas, en su mayoría no electorales.

Los antecedentes citados en relación con las variables "sociopsicológicas" (eficacia política, interés político, conocimiento político y confianza política), los valores C/I y las actitudes ideológicas permiten la formulación de un conjunto de hipótesis que se ponen a prueba en el presente estudio que pretende establecer qué conjunto de variables permite discriminar entre participantes y no participantes de acuerdo a cuatro dimensiones de la PP (Sorribas, 2014). 
De acuerdo a los resultados de Arlin (2006) sobre la asociación positiva del RWA con las actitudes hacia la violencia - que hacen que este constructo sea considerado como una de las tendencias que constituye una amenaza a la democracia- es de esperar mayores niveles de RWA en quienes no recurran a repertorios participativos institucionalizados y de carácter colectivo.

La relación de la SDO-OD con el RWA ha sido ampliamente documentada en la literatura especializada. Por lo tanto, es de esperar también altos niveles de SDO-OD (asociados con altos niveles de RWA) en quienes no participen institucionalmente y de modo colectivo.

En relación a los valores, se ha reportado que los individualistas son más proclives a pertenecer a grupos exclusivos (Shulruf, Hattie \& Dixon, 2007) en comparación con los más colectivistas. Los colectivistas se identifican como miembros de un grupo al que pertenecen y son más propensos a internalizar las metas del grupo y sus valores y dar a estos mayor prioridad (Shulruf et al., 2007). A partir de estos hallazgos es de esperar que los participantes con valores colectivistas se involucren en acciones de contacto con organizaciones sociales e instituciones públicas.

Los focos de las escalas que evalúan individualismo y colectivismo políticos llevan a proponer las siguientes hipótesis: quienes acuerden en mayor medida con el colectivismo político, tenderán a involucrarse en las diferentes dimensiones de la PP. Y los que se orienten más por el individualismo político se involucrarán en acciones demandadas por partidos políticos.

De acuerdo a los antecedentes sobre eficacia política, es esperable que se asocie a amplios repertorios participativos, desde los más pasivos hasta los más activos. Por ello, quienes participen en cualquiera de las dimensiones de la PP reportarán niveles más altos en esta variable. França (1995) constata que se logran mejores predicciones utilizando medidas específicas de eficacia. Morrell (2003) pudo establecer la confiabilidad de indicadores que fueron diseñados para evaluar más específicamente este constructo dando lugar a una escala de eficacia política participativa. En base a esto, es de esperar que quienes reporten niveles más altos en ella sean quienes reporten actividad en las dimensiones participativas más afines a las acciones contempladas en el instrumento. 
La asociación del conocimiento político con modalidades convencionales como las campañas electorales, donar dinero y el establecimiento de contactos con funcionarios públicos está ampliamente reportada (McAtee \&Wolak, 2011). Esto puede explicarse por el contenido de los ítems de las escalas diseñadas para evaluar este constructo. En ocasiones su contenido se orienta a una perspectiva formal de la política, privilegiando los roles de instituciones y funcionarios políticos más salientes en el contexto local, lo cual implicaría un conocimiento más cercano al repertorio más afín a las reglas de juego y de los actores claves en el sistema político institucionalizado. Por ello, es de esperar un mayor conocimiento político en quienes reporten participación de tipo partidaria - electoral.

La literatura sugiere una relación directa y positiva del interés político con muy variados repertorios participativos: participación social y política (Mannarini et al., 2008); participación institucionalizada (Marien et al., 2010) y participación no electoral (Klesner, 2007; Robnett, 2007). De allí que puede esperarse que quienes reporten mayores niveles de interés político participen en cada una de las cuatro dimensiones del repertorio participativo local.

Por último, es esperable que niveles altos de confianza política se asocien al activismo electoral y a acciones de apoyo al gobierno (Grossi \& Ovejero, 1994). En cambio niveles bajos o insatisfacción con políticas gubernamentales se asociaría con participantes que recurren a acciones contestatarias o de protesta (John et al., 2011; Van der Meer \& Van Ingen, 2009; Van der Meer et al., 2009).

\section{Método}

\section{Participantes}

La muestra estuvo conformada por 450 ciudadanos residentes en la ciudad de Córdoba, Argentina, mayores de 18 años (52\% de mujeres) y que se encontraban en un rango de edad entre 18 y 85 ańos $\left(M_{\text {edad }}=40.5 D E_{\text {edad }}=16.98\right)$. El muestreo fue probabilístico y se realizó 
de acuerdo a la metodología de probabilidad proporcional al tamaño. Esta técnica proporciona una muestra probabilística (con características de aleatoriedad y representatividad) y es útil cuando las unidades de muestreo varían considerablemente en tamaño (por ej.: el tamaño poblacional que cada uno de los radios censales posee) ya que es posible asegurarse que aquellos lugares con mayor tamaño tengan la misma probabilidad de ser seleccionados que los lugares con menor tamaño (y viceversa) (Kish, 1965). El nivel educativo fue mayoritariamente "universitario incompleto" (25\%), seguido de "secundario completo" (23\%). La mayoría de los hogares (54\%) corresponde a un nivel socioeconómico entre "medio bajo" y "bajo superior" (28\% y $26 \%$ respectivamente), y la mayoría de los encuestados se encontraba "ocupada" (55\%) y un 14\% se encontraba "desocupado".

\section{Instrumentos}

En la primera sección del cuestionario se recopilaron datos sociodemográficos (género, edad y nivel socioeconómico). En las otras secciones se incluyeron las siguientes escalas:

Individualismo y colectivismo. Medida a través de la versión traducida de Shulruf et al. (2007) de la Escala Auckland de Individualismo Colectivismo. Autoinforme de 22 ítems que mide valores individualistas y colectivistas. Formato de respuesta tipo Likert con 6 opciones, desde "nunca" hasta "siempre". Mediante análisis factorial se identificaron dos factores: Individualismo (por ej.: "Es importante para mí actuar como una persona independiente") y colectivismo (por ej.: "Considero las opiniones de mis amigos antes de realizar acciones importantes"). Los análisis de confiabilidad en base a la muestra resultaron aceptables: $\alpha=.83$ y $\alpha=.81$ respectivamente.

Individualismo-colectivismo políticos. A través de una escala diseñada por Sorribas (2012) con 11 ítems evaluados por una escala Likert de 6 puntos, desde "fuertemente en desacuerdo" a "fuertemente de acuerdo". Mediante análisis factorial se identificaron dos factores: colectivismo político (por ej.: "Las injusticias del país te llevan a pensar que no se resuelven solas sino participando junto a otros") e individualismo 
Político (por ej.: "Si en política uno encuentra una oportunidad hay que aprovecharla por más que pueda perjudicar a otros"). Los análisis de confiabilidad en base a la muestra resultaron aceptables: $\alpha=.80 \mathrm{y}$ $\alpha=.60$ respectivamente. Esta escala se incluyó para especificar la evaluación de los valores I/C en relación con el dominio político.

Orientación a la dominancia social. Medida con la versión traducida de Weber y Federico (2007). Cuenta con 8 ítems puntuados mediante una escala Likert de 6 puntos ("fuertemente en desacuerdo" a "fuertemente de acuerdo") y que evalúan dos factores: orientación hacia la igualdad grupal (OI: por ej.: "Debemos esforzarnos por hacer que los ingresos sean tan iguales como sea posible") y orientación hacia la dominancia grupal (OD: por ej.: "Los grupos inferiores deben permanecer en su lugar"). Los análisis de confiabilidad en base a la muestra resultaron aceptables: $\alpha=.74$ y $\alpha=.71$, respectivamente. El mayor puntaje indica mayor orientación hacia el polo de la SDO: Igualdad o dominancia.

Autoritarismo de derecha. Versión traducida de Weber y Federico (2007). Cuenta con 9 ítems evaluados a través de una escala Likert de 6 puntos ("fuertemente en desacuerdo" a "fuertemente de acuerdo"). Mediante análisis factorial se identificaron dos factores: orientación Autoritaria (OA: por ej.: "En estos tiempos difíciles, las leyes tienen que ser ejecutada sin piedad, sobre todo cuando se trata de los agitadores y revolucionarios que están desbordando las cosas") y orientación democrática (OD: por ej.: "El lugar de una mujer debe estar donde ella quiere que esté. Los días en que las mujeres eran sumisas a sus maridos y a las convenciones sociales pertenecen estrictamente al pasado"). Los análisis de confiabilidad en base a la muestra resultaron aceptables: $\alpha=.84$ y $\alpha=.48$. En ambas un mayor puntaje indica un mayor nivel de autoritarismo.

Eficacia Política. Medida a través de la versión de la Escala de Brussino et al. (2011) con 7 ítems evaluados con una escala Likert de 6 opciones, desde "fuertemente en desacuerdo" a "fuertemente de acuerdo" (por ej.: "Siento que podría realizar un trabajo en un puesto político tan bien como cualquier otra persona"). El análisis de confiabilidad sobre la base de la muestra reportó un resultado aceptable $(\alpha=.75)$. 
Eficacia politica participativa. Escala diseñada por Sorribas (2012) con 7 ítems evaluados mediante una escala Likert de 6 opciones, desde "fuertemente en desacuerdo" a "fuertemente de acuerdo" (por ej.: "Las consultas populares sirven para controlar y limitar la relación del Estado con las empresas privadas"). El análisis de confiabilidad en base a la muestra reportó un resultado aceptable $(\alpha=.71)$.

Conocimiento político. Medida a través del Índice de Conocimiento Político, que integra la escala de Brussino et al. (2008) y los ítems desarrollados por Sorribas (2012). Cuenta con 11 ítems (por ej.: “¿Cuál es el partido con más miembros en el Poder Legislativo provincial?", "En la ciudad de Córdoba: ¿̨un grupo de ciudadanos puede promover un Referéndum?”). Cada respuesta sustantiva se puntuó utilizando una escala de 4 posiciones ( $0=$ No sabe a $4=$ completamente correcta). El análisis de confiabilidad sobre la base de la muestra reportó un resultado aceptable $(\alpha=.77)$.

Confianza politica. Escala diseñada por Sorribas (2012) con 14 ítems evaluados con una escala Likert de 6 opciones (por ej.: “¿Cuán confiable es para Ud. el Concejo Deliberante de Córdoba”?). El análisis de confiabilidad en base a la muestra reportó un resultado aceptable $(\alpha=.89)$.

Interés político. Diseñada por Brussino et al. (2011) con 9 ítems evaluados mediante una escala Likert de 6 opciones, desde "fuertemente en desacuerdo" a "fuertemente de acuerdo". (por ej.: "Me interesa averiguar cómo funcionan los partidos políticos"). El análisis de confiabilidad en base a la muestra reportó un resultado aceptable $(\alpha=.92)$.

Participación politica. Escala diseñada por Sorribas (2014), con cuatro factores: participación electoral - de campaña (con 7 ítems; por ej.: "Obtener avales para un partido político", "Asistencia a reuniones de equipos técnicos de un partido político"); participación de contacto (7 ítems; por ej.: "Contacto con centros vecinales"); participación contenciosa (6 ítems; por ej.: "Cortes de calles-rutas") y participación para influenciar las decisiones del Poder Legislativo (4 ítems; por ej.: "acciones directas por la Ley matrimonio igualitario"). Los análisis de confiabilidad reportados por Sorribas (2014) resultaron aceptables 
(primera dimensión: $\alpha=.81$; segunda dimensión: $\alpha=.71$; tercera dimensión: $\alpha=.69$ y cuarta dimensión: $\alpha=.70)$. Para el presente estudio cada acción fue valorada dicotómicamente $(1=$ la hizo y $0=$ no la hizo). En función de esa evaluación se determinó para cada sujeto si participó o no en cada una de las cuatro dimensiones de la escala ( 1 = Sí participó y $0=$ No participó).

\section{Procedimiento}

El procedimiento se inició con la elección, de manera aleatoria, de las unidades primarias (en este caso representadas por radios censales) y dentro de estas últimas se seleccionaron las manzanas — con probabilidades iguales - para, por último, elegir aleatoriamente viviendas al interior de cada manzana. De estas, solo se seleccionó una persona para ser encuestada y el criterio de inclusión fue ser ciudadano residente en Córdoba y tener 18 años o más. En cada manzana se completaron 8 casos.

Por cada vivienda un residente prestó su consentimiento — previa información sobre los objetivos de la investigación y la confidencialidad de los datos - para responder la encuesta. El cuestionario fue administrado por un encuestador debidamente entrenado, de manera individual, en el hogar y la duración fue de aproximadamente 45 minutos. Se controló el relevamiento por la variable género, tratando de encuestar en cada punto muestra (manzana) de la ciudad una cantidad equivalente de mujeres y varones.

\section{Análisis de datos}

Los datos se analizaron con SPSS .17. Se utilizó la técnica de análisis discriminante $(\mathrm{AD})$ de dos grupos, debido a que la variable criterio es dicotómica y las variables independientes son métricas. Cada una de las dimensiones de la participación política (Sorribas, 2014) fue considerada como variable criterio y se estableció con categorías mutuamente excluyentes y colectivamente exhaustivas: el valor " 0 " correspondió a "no participación" en dimensión alguna de las cuatro que conforman 
el repertorio participativo. El valor "1" correspondió a "sí participó" en la dimensión analizada. En base a este criterio la muestra en cada uno de los análisis fue diferente en tamaño y menor a la muestra total $(\mathrm{N}=450)$. Para la aplicación del AD se siguió el método stepwise para el ingreso de las variables independientes. Para cada dimensión se procedió al examen de la función discriminante para determinar la importancia relativa de cada una de las variables independientes para discriminar entre los dos grupos establecidos para cada dimensión de la PP, a través de los pesos discriminantes estandarizados. Luego, a partir de la función discriminante identificada se inspeccionó la distancia entre los grupos a través de los centroides. Por último, se analizó en qué grado es acertada la predicción en base a los resultados de la clasificación para los dos grupos en relación a cada dimensión de la PP.

\section{Resultados}

Dado que el análisis discriminante se realizó sobre dos grupos poblacionales (participantes - no participantes), en cada caso se obtuvo una única función discriminante.

\section{Dimensión partidaria-de campaña}

Para esta dimensión se reportaron un total de 85 casos (19\% de la muestra) que informaron haber participado a través de alguno de sus acciones. El grupo de "no participantes" en acción alguna quedó conformado por 197 sujetos.

Los resultados de la prueba $\mathrm{M}$ de $\operatorname{Box}(M=23.40 F=2.30 p=.13)$ no reflejan diferencias significativas entre las matrices de covarianzas de los dos grupos (no participa - participa). Por lo tanto, se cuenta con un criterio sólido para la aplicación del análisis discriminante. El análisis por el método stepwise permitió establecer que cuatro variables conforman la función discriminante: (1) interés político, (2) RWA-OD, (3) eficacia política participativa y (4) valor: colectivismo. La Tabla 1 ilustra la función discriminante. El coeficiente de Lambda de Wilks 
resultó significativo $\left(\chi^{2}=65.609 ; p=.00\right)$, por lo que se rechaza la hipótesis nula de que los grupos comparados tienen promedios iguales en las cuatro variables discriminantes. A su vez, el coeficiente de correlación canónica resulta adecuado $(r=.458)$, mostrando un modelo válido para discriminar entre los dos grupos.

\section{Tabla 1}

Coeficientes estandarizados de la función discriminante canónica

\begin{tabular}{lc}
\hline \multicolumn{1}{c}{ Partidaria - de campaña } & Función 1 \\
\hline Interés político & .74 \\
Eficacia política participativa & .42 \\
RWA- OD & -.44 \\
Valor colectivismo & -.37 \\
\hline
\end{tabular}

Sobre la base de los valores de los centroides reportados en la Tabla 2 puede afirmarse que los grupos se separan adecuadamente.

\section{Tabla 2}

Funciones en los centroides de los dos grupos

\begin{tabular}{lc}
\hline \multicolumn{1}{c}{ Partidaria - de campaña } & Función 1 \\
\hline No participa & -.338 \\
Si participa & .783 \\
\hline $\begin{array}{l}\text { Nota: Funciones discriminantes canónicas no tipificadas evaluadas en las } \\
\text { medias de los grupos }\end{array}$
\end{tabular}

Por último, los resultados de la Tabla 3 sobre la clasificación de los dos grupos indican que se predice con acierto el $77 \%$ de los casos. Este valor es levemente superior al del criterio de máxima aleatoriedad (70\%) que determina la clasificación aleatoria basándose en el tamaño muestral del grupo más grande. De todos modos este $77 \%$ no cumple el criterio de precisión clasificatoria que exige un cuarto por encima 
del porcentaje obtenido por aleatoriedad ${ }^{3}$ (Hair, Anderson, Tatham $\&$ Black, 1999). Se pronostica correctamente a los que "Sí participan" en un porcentaje bajo $(43.5 \%)$ y en un porcentaje más aceptable $(91 \%)$ a los que "No participan".

\section{Tabla 3}

Porcentaje de casos correctamente clasificados para la dimensión partidariade campaña ${ }^{a}$

\begin{tabular}{|c|c|c|c|c|c|}
\hline \multirow{2}{*}{\multicolumn{2}{|c|}{ Clasificación }} & \multirow{2}{*}{$\begin{array}{l}\text { Partidario } \\
\text { electoral }\end{array}$} & \multicolumn{2}{|c|}{$\begin{array}{l}\text { Grupo de pertenencia } \\
\text { pronosticado }\end{array}$} & \multirow[t]{2}{*}{ Total } \\
\hline & & & No participa & Sí participa & \\
\hline \multirow{4}{*}{ Original } & Recuento & No participa & 179 & 18 & 197 \\
\hline & \multirow{3}{*}{$\%$} & Si participa & 48 & 37 & 85 \\
\hline & & No participa & $90.9 \%$ & $9.1 \%$ & $100 \%$ \\
\hline & & Si participa & $56.5 \%$ & $43.5 \%$ & $100 \%$ \\
\hline
\end{tabular}

Nota: ${ }^{a}$ Clasificados correctamente el $77 \%$ de los casos agrupados originales

Sobre la base de estos resultados se pudo confirmar la hipótesis que establecía que quienes puntuaran más alto en la escala de RWA no participarían en acciones institucionalizadas como las relativas a la dimensión partidaria - de campańa. A la par, tal como se esperaba el interés político es mayor en quienes participan. Las hipótesis relativas al constructo SDO, a los valores colectivismo político e individualismo político y a la eficacia política no se confirmaron.

\section{Dimensión de contacto con organizaciones y/o referentes politicos}

En relación con esta dimensión se observó que quienes optaron por estas modalidades participativas constituyen el 35\% de la muestra y han efectuado un total de 322 acciones. El grupo de participantes incluyó a 153 sujetos y el de no participantes a 197. 
El $p$-valor de la prueba $M$ de $\operatorname{Box}(M=16.90 F=1.67 p=.12)$ no refleja diferencias significativas entre las matrices de covarianzas de los dos grupos y por lo tanto, se puede aplicar el análisis discriminante.

Las variables independientes incluidas en la función discriminante son cuatro: (1) interés político, (2) colectivismo político, (3) colectivismo y (4) RWA-OD. La Tabla 4 ilustra la función discriminante para esta dimensión. El coeficiente de Lambda de Wilks resultó significativo $\left(\chi^{2}=80.61 ; p=.000\right)$. A su vez, el coeficiente de correlación canónica resultó adecuado $(r=.456)$, mostrando un modelo válido para discriminar entre los dos grupos.

\section{Tabla 4}

Coeficientes estandarizados de la función discriminante canónica

\begin{tabular}{lc}
\hline \multicolumn{1}{c}{ Repertorio de contacto } & Función $\mathbf{1}$ \\
\hline Interés político & .61 \\
Colectivismo político & .49 \\
Valor colectivismo & -.37 \\
RWA - OD & -.35 \\
\hline
\end{tabular}

En la Tabla 5 se constata que los centroides de la función discriminante para cada grupo los separan adecuadamente.

\section{Tabla 5}

Funciones en los centroides de los dos grupos

\begin{tabular}{lc}
\hline \multicolumn{1}{c}{ Repertorio de contacto } & Función 1 \\
\hline No participa & -.450 \\
Si participa & .580 \\
\hline Nota: Funciones discriminantes canónicas no tipificadas evaluadas en las \\
medias de los grupos
\end{tabular}


Los resultados de la Tabla 6 sobre la clasificación para los dos grupos indican que se predice con acierto el $71 \%$ de los casos. Este valor es superior al del criterio de máxima aleatoriedad (56.3\%). Tal porcentaje $(71 \%)$ supera levemente al criterio de precisión clasificatoria (Hair et al., 1999) establecido para este repertorio en 70.3\%. Así, se pronostica correctamente a los que Sí participan en un porcentaje bajo (58.2\%) y en un porcentaje más aceptable (80.2\%) a los que No participan.

\section{Tabla 6}

Porcentajes de casos correctamente clasificados para la dimensión de contacto $^{a}$

\begin{tabular}{cccccc}
\hline \multirow{2}{*}{ Clasificación } & \multirow{2}{*}{$\begin{array}{c}\text { Repertorio } \\
\text { de contacto }\end{array}$} & \multicolumn{2}{c}{$\begin{array}{c}\text { Grupo de pertenencia } \\
\text { pronosticado }\end{array}$} & \multirow{2}{*}{ Total } \\
\cline { 3 - 5 } & & Si participa & No participa & \\
\hline \multirow{3}{*}{ Original } & \multirow{2}{*}{ Recuento } & No participa & 158 & 39 & 197 \\
& & Si participa & 64 & 89 & 153 \\
& & No participa & 80,2 & 19.8 & 100 \\
& & Si participa & 41.8 & 58.2 & 100 \\
\hline
\end{tabular}

Nota: ${ }^{a}$ Clasificados correctamente el $71 \%$ de los casos agrupados originales.

En relación a las hipótesis planteadas se pudo confirmar que los que reportan mayor nivel de RWA no participan y que los que puntúan más alto en colectivismo político sí participan. La relación esperada entre la SDO, la eficacia política y esta dimensión no se confirmó. El interés político contribuye a discriminar los grupos en el sentido esperado. En relación con el valor colectivismo se observó una relación inversa a la esperada, quienes son menos colectivistas participan mediante este repertorio de acciones. Por último, la eficacia política participativa no quedó incluida en la función discriminante como era de esperar. 


\section{Dimensión contestataria y reivindicativa y dimensión orientada a influir las decisiones del Poder Legislativo}

Un 32\% de los integrantes de la muestra (144 sujetos) participó mediantes acciones directas y más orientadas a estrategias reivindicativas en lo laboral y con un mayor nivel contestatario para expresar su involucramiento político. En total protagonizaron 268 acciones. Los no participantes en acción alguna fueron 197.

Por otra parte se constató que un $23 \%$ de los integrantes de la muestra poblacional recurrió a acciones correspondiente a la dimensión orientada a influir sobre las decisiones del Poder Legislativo, protagonizando un total de 196 acciones. Los participantes efectivos fueron 105 sujetos y los no participantes 197.

$\mathrm{Al}$ revisar el supuesto de homocedasticidad, la prueba $\mathrm{M}$ de Box reportó valores $(M=43.55 F=1.669 p=.00$ y $M=65.362 \mathrm{~F}=3.037$ $p=.00$, respectivamente) que indican que no existe igualdad de las matrices de covarianza entre los dos grupos y por lo tanto no correspondía efectuar el $\mathrm{AD}$.

\section{Participación mixta}

Por último, se realizó un análisis estableciendo una variable criterio diferente: el valor "0" correspondió a "no participación" en modalidad alguna de las cuatro dimensiones y el valor " 1 " a participación mixta (dos o más dimensiones de la PP). El valor de la prueba $\mathrm{M}$ de Box $(M=51.320 F=2.397 p=.00)$ también indica que no se confirma la existencia de la igualdad de las matrices de varianzas y covarianzas de las variables analizadas en los dos grupos.

A modo de síntesis de los resultados expuestos se puede destacar que: (a) cinco variables permiten discriminar entre participantes y no participantes en dos de las cuatro dimensiones de la PP; (b) tres de estas variables han sido incluidas en las dos funciones discriminantes, mostrando iguales signos (interés político, el RWA-OD y colectivismo); (c) una de estas variables correspondió a las actitudes ideológicas (RWA-OD), otras dos fueron variables "sociopsicológicas" ampliamente 
referenciadas en la literatura (interés político y eficacia política participativa) y otras dos correspondieron a valores: colectivismo y colectivismo político; (d) la eficacia política participativa fue incluida en la función discriminante de la dimensión partidaria-de campaña y registró un peso discriminante positivo; (e) para la dimensión de contacto se supera levemente el criterio de precisión clasificatoria; (f) en las dos dimensiones las funciones discriminantes establecidas permitieron una mejor clasificación del grupo que No participa; (h) para las dos dimensiones los centroides permitieron separar adecuadamente a los grupos; (i) los resultados del análisis de la igualdad de las matrices de covarianza entre los dos grupos indicaron que correspondía realizarse el AD en el caso de la dimensión que presentaba el menor grupo de participantes (dimensión electoral - de campańa con $\mathrm{N}=85$ ) y en la dimensión que presentaba el mayor grupo de participantes (dimensión de contacto con $\mathrm{N}=153$ ).

\section{Discusión}

Un estudio reciente ha identificado cuatro dimensiones de la PP (Sorribas, 2014) que evidencian la amplitud del repertorio participativo de los ciudadanos de Córdoba, Argentina. En función de esta evidencia sobre la multidimensionalidad de la PP, en el presente trabajo se procedió a identificar qué variables permiten discriminar entre participantes efectivos y no participantes. Los resultados indican que tanto para una dimensión electoral como para una no electoral, un subconjunto de las variables evaluadas permite discriminar ambos grupos. A su vez, este subconjunto incluye variables menos estudiadas en relación con dimensiones no electorales de la PP como lo son el RWA-OD, el colectivismo y el colectivismo político. En función de estos hallazgos se puede interpretar el aporte específico hecho por estas variables en la discriminación de esas dos dimensiones participativas.

En el marco de la multidimensionalidad de la PP, la dimensión partidariade campańa puede constituir un repertorio intermitente que es modelado por los canales o agencias de movilización (Norris, 2002; 
Teorell, 2003; Vráblíková, 2010) que incentivan la participación. Esa motivación externa podría combinarse con el interés político en tanto constructo relativo a la motivación interna para este tipo de acción. Así, se explicaría su contribución a la discriminación entre participantes y no participantes. Este efecto es ampliamente referenciado en los antecedentes sobre repertorios similares (Klesner, 2007; Mannarini et al., 2008; McAtee \& Wolak, 2011; Rodríguez et al., 1993; Whiteley \& Seyd, 2002; entre otros) y es consistente con otros estudios realizados sobre la misma población (Brussino, Rabbia \& Sorribas, 2009).

Para entender el aporte del valor colectivismo a esta dimensión se debe considerar que este constructo captaría principalmente la subdimensión "consejo" y refiere a vínculos endogrupales con la familia, los amigos y personas cercanas y en menor medida captaría la dimensión “armonía”(Shulruf et al., 2007). Entonces quienes sean más autónomos o independientes respecto de esos vínculos, serían los que participen del espacio público que caracteriza a este repertorio partidario de campańa.

Como se esperaba quienes se perciben con mayor sentido de eficacia política participativa se involucran políticamente mediante este repertorio. La escala que evaluó este constructo se diseñó considerando los aportes de França (1995), Morrell (2003) y Wollman y Stouder (1991) y enfatizó la evaluación de resultados efectivos que se alcanzarían mediante la participación colectiva principalmente mediante canales institucionalizados y a través de mecanismos de la democracia directa y participativa propios de Argentina. Esto permite interpretar la contribución de esta variable al discriminar participantes de no participantes en acciones enmarcadas dentro de las instituciones partidarias. De esta manera contamos con evidencia respecto de la articulación entre dimensiones electorales y no electorales de la PP. En tal sentido el involucramiento en partidos y campañas electorales haría que la percepción de eficacia relativa a estos nuevos mecanismos de participación sea mayor. En futuras indagaciones se debería comprobar si quienes se vinculan a los partidos y/o campañas también lo hacen con las instituciones de democracia directa y participativa que tienen a disposición en su ámbito político inmediato. 
En relación con las actitudes ideológicas se pudo establecer que quienes tienen creencias más autoritarias no se involucran en acciones vinculadas al sistema partidario y las campañas electorales. Este hallazgo no es susceptible de una interpretación ajustada a la mayoría de los antecedentes que han evaluado la relación entre RWA y el comportamiento electoral, ya que en ellos se suelen considerar las preferencias partidarias de acuerdo al posicionamiento ideológicos de los partidos políticos (ver Duckitt \& Fisher, 2003). De todos modos el efecto negativo sobre esta dimensión de la PP puede entenderse por la asociación del RWA con valores sociales como la tradición, la conformidad, la seguridad y la ortodoxia (Crowson et al., 2005; Duckitt \& Sibley, 2009); con el conservadurismo cultural (Van Hiel, Cornelis, Roets \& De Clercq, 2007), y en el campo político por su asociación con el conservadurismo político focalizando sobre la resistencia al cambio (Jost, Glaser, Kruglanski \& Sulloway, 2003; Mavor et al., 2010). De ser adecuada esta interpretación, quienes se involucran con partidos y en campañas no orientarían su acción por dichos valores. En futuras indagaciones podría confirmarse esta relación indagando las preferencias partidarias de estos activistas, la percepción del posicionamiento ideológico de esos partidos y si se corresponden con opciones partidarias más tradicionales dentro del sistema partidario de Argentina.

Por otra parte, la dimensión de contacto integra acciones que en otros estudios son consideradas como sociales o comunitarias y puede constituir una forma de involucramiento que vincula a las personas específicamente con las políticas públicas implicando un uso activo de ciertos beneficios sociales y/o un fortalecimiento del capital social comunitario - barrial (Velásquez \& Martínez, 2004). Sin embargo, el interés político - cuyo instrumento de medición es más afín a los procesos electorales - alcanzó un alto peso discriminante. Así, este factor motivacional sería transversal a las dos dimensiones participativas demostrando su aporte en relación con una dimensión no electoral de la PP.

Respecto del aporte del valor colectivismo a esta segunda dimensión, corresponde hacer la misma interpretación anterior. En tal sentido 
los más colectivistas —en relación a la dependencia de endogrupos familiares y de amigos cercanos - no se involucrarían con el espacio público que caracteriza a la participación de contacto.

El constructo colectivismo político —a diferencia del colectivismo - refiere más a una concepción de interdependencia, es decir que los grupos se unen entre sí y obligan a las personas (Oyserman, 1993). A su vez, la referencia a endogrupos de este constructo abarca diversos tipos de colectivos y - consecuentemente- refiere a un mayor rango de valores, actitudes y comportamientos (Bourgeois, 2002; Hui, 1988). Esta conceptualización permite entender su poder discriminante en esta dimensión que se orienta hacia la mediación de diversos tipos de organizaciones con diferentes grados de formalización.

La contribución del RWA-OD indica que quienes se sitúan como menos autoritarios se involucran en acciones de contacto. Esta dimensión no se enmarca exclusivamente en mecanismos de democracia representativa, por lo cual se requiere de una interpretación adicional sobre el significado que adquiere aquí esta actitud ideológica. En tal sentido, cabría esperar que quienes cuenten con una menor orientación autoritaria se vinculen con organizaciones o contacten a referentes de la política sin el propósito de fortalecer aquellos componentes agresivos de las autoridades que pueden dirigirse contra individuos o grupos no convencionales (Walter et al., 1996); y consecuentemente no asumirían una posición sumisa antes dichas autoridades (Altemeyer, 1981, 1988, 1996; citado en Van Hiel et al., 2007). Los objetivos que orientan estas acciones de contacto (ej.: demanda de servicios públicos; colaborar en acciones sociales y en prestaciones para cubrir necesidades básicas) avalan una interpretación en esta dirección. En tal sentido, esta dimensión de contacto vincula a los ciudadanos con diferentes organizaciones con el propósito de hacer un uso activo de ciertos beneficios sociales que no estarían siendo garantizados "desde arriba" a través de políticas públicas eficaces. Si la RWA opera como una ideología justificante del sistema (Jost \& Hunyady, 2002), se reconoce en estos resultados que los menos autoritarios son quienes cuestionan cierto status quo en relación a políticas públicas ineficientes o injustas. 
En relación con el propósito de evaluar el aporte que realizan las actitudes ideológicas y los valores a la discriminación entre participantes y no participantes en las distintas dimensiones participativas, es posible sostener que se avanzó en identificar su contribución en dos de las cuatro dimensiones de la PP analizada. Si bien queda pendiente establecer si tales constructos se asocian a otras dos dimensiones (la orientada a influir las decisiones del Poder Legislativo y la contenciosa-reivindicativa), se estableció que contribuyen a discriminar entre participantes y no participantes en dos dimensiones bien diferenciadas. Una de ellas corresponde a la más tradicional dimensión electoral —vínculo con partidos políticos y procesos de campaña-, mientras la otra refiere a modalidades por fuera del sistema partidario que intentan un uso activo de ciertos beneficios sociales no garantizados desde arriba.

En términos temporales también se distinguen ya que remiten a momentos disímiles de la política. La dimensión partidaria - de campańa está regulada desde arriba e implica acciones dentro de un repertorio intermitente que es modelado por los canales o agencias de movilización (Norris, 2002; Teorell, 2003; Vráblíková, 2010) que incentivan a la participación. En cambio la dimensión de contacto es regulada desde debajo de acuerdo a las evaluaciones y percepciones de los ciudadanos en torno a bienes públicos y beneficios sociales.

$Y$ en relación al tipo de organizaciones también ambas dimensiones están diferenciadas. La partidaria - de campaña implica el vínculo con partidos políticos es decir con asociaciones políticas tradicionales (Morales, 2009), en cambio la dimensión de contacto refiere a las organizaciones políticas caracterizadas por la ausencia de un vínculo representativo con sectores específicos de la población y sus demandas —es decir los problemas que representan- serían más universalistas en sus objetivos (Morales, 2009).

El aporte de constructos ampliamente referenciados como el interés político y la eficacia política participativa también ha sido corroborado para estas dos dimensiones que se diferencian entre sí. El primero formando parte de ambas funciones discriminantes y dando cuenta así de la importancia del factor motivacional para dos repertorios políticos 
diferenciados. De todos modos el interés político aporta un mayor peso discriminante en la dimensión partidaria - de campańa en comparación con la dimensión de contacto. Esto puede deberse a que el contenido de los ítems de la escala de interés político (Brussino et al., 2011) refieren mayormente a una concepción de lo político restringida al sistema representativo y partidario, es decir a una dimensión electoral de la PP. Por su parte la eficacia política participativa solo demostró su capacidad discriminante en relación con la dimensión partidaria - de campaña, corroborando lo sostenido por França (1995), Morrell (2003) y Wollman y Stouder (1991) en términos del diseño del instrumento. Este hallazgo permite sostener la importancia de seguir desarrollando escalas que consideren la especificidad situacional al evaluar los sentimientos de eficacia asociados a distintos tipos de acciones políticas. El carácter genérico del sentimiento de eficacia evaluado por la escala de eficacia política (Brussino et al., 2011) puede ser la razón por la que no aporte a la discriminación entre participantes y no participantes en dimensiones específicas de la PP.

Como se ha evidenciado fue factible aplicar el $\mathrm{AD}$ a dos de las cuatro dimensiones de la participación política. Una interpretación posible para la limitación observada en relación con las dimensiones "participación contenciosa" y "participación para influenciar las decisiones del Poder Legislativo" puede referirse a que la mayoría de los sujetos participantes en ellas reportaba a su vez participación en la dimensión de contacto. Así, el 65\% de quienes se involucraron en acciones contenciosas también afirmó participar del repertorio de contacto. El mismo patrón de involucramiento se observó para el 57\% de los participantes que realizaron acciones orientadas a influenciar las decisiones del Poder Legislativo. Futuros análisis sobre la participación mixta (en dos, tres y cuatro dimensiones) permitirían valorar la validez de dicha interpretación.

Por último, si bien el $\mathrm{AD}$ permitió discriminar los grupos para dos dimensiones de la PP, la mejor precisión clasificatoria fue en ambos casos para el grupo de los "no participantes". Tal resultado puede obedecer al carácter más homogéneo de este grupo. Sus integrantes no 
expresan involucramiento alguno en el ámbito político. Y esto se observó a pesar de la amplitud de la escala que se empleó para valorar la participación política en esta población. Por ello, pueden experimentar un similar desinterés por la política y un menor sentimiento de eficacia participativa. En cambio, el grupo de "participantes" se definió por su involucramiento en al menos una acción de las incluidas en las dimensiones de dicha escala. Consecuentemente este grupo es más heterogéneo en su conformación. Integra tanto a sujetos que pueden haber recurrido a una única acción correspondiente a una dimensión, como a otros que pueden haber realizado hasta siete acciones dentro de la misma dimensión. A su vez, como ya se indicó muchos sujetos han reportado una participación mixta (en dos o más dimensiones). Tal heterogeneidad en términos comportamentales puede implicar variación en los sentimientos de eficacia, en el Interés Político, en los valores y en las orientaciones autoritarias o democráticas asociadas al RWA. De allí, que sea menos precisa la clasificación de este grupo en función del subconjunto de variables con capacidad discriminante.

La identificación de un conjunto de variables que discriminan entre quienes participan y quienes no participan considerando tanto una dimensión electoral como una no electoral de la participación política en Córdoba, Argentina permitió aportar evidencia de la relación del RWA con variados comportamientos políticos, complementando los antecedentes que lo vinculaban con las preferencias partidarias en términos de posicionamientos ideológicos de estos actores de la política. A su vez, la relación de dos valores diferenciados (colectivismo y colectivismo político) con la participación política constituye un aporte a la amplia literatura sobre los predictores del involucramiento político. Por último, la medida participativa de la eficacia política demostró su contribución, aportando evidencia de la relevancia de contar con escalas que impliquen mayor especificidad situacional en los sentimientos de eficacia. 


\section{Referencias}

Arlin, J. B. (2006). The relationship between right-wing authoritarianism and attitudes toward violence: Further validation of the attitudes toward violence scale. Social Behavior and Personality, 34(8), 923-926. https://doi.org/10.2224/sbp.2006.34.8.923

Bolzendahl, C. \& Coffé, H. (2010). Diverse democracies: Citizenship beliefs and political participation across three geopolitical regions.

UC Irvine: Center for the Study of Democracy.

Bourgeois, D. Y. (2002). The politics and values of individualist and collectivists: A cross cultural comparison. Disertación para optar al grado de Doctor, University of Maine. UMI N 3074225.

Brussino, S., Medrano, L., Sorribas, P. M. \& Rabbia, H. (2011). Young adults' knowledge of politics: Evaluating the role of socio-cognitive variables using structural equations. The Spanish Journal of Psychology, 14(1), 183-194. https://doi.org/10.5209/ rev_SJOP.2011.v14.n1.16

Brussino, S., Rabbia, H. \& Sorribas, P. M. (2008). Una propuesta de categorización de la participación política de jóvenes cordobeses. Psicología Política, 8(16), 285-304.

Brussino, S., Rabbia, H. \& Sorribas, P. M. (2009). Perfiles sociocognitivos de la participación política de los jóvenes. Interamerican Journalof Psychology, 43(2), 279-287.

Brussino, S., Sorribas, P. M., Rabbia, H. H. \& Imhoff, D. (2013). Enfrentando los desafios em la evaluación de La participación política: aportes a la discusión sobre indicadores y escalas. Polis [En línea], 35, 1-17. Recuperado de: http://polis.revues.org/9117

Cárdenas, M., Meza, P., Lagues, K. \& Yañez, S. (2010). Adaptación y validación de la escala de orientación a la dominancia social (SDO) en una muestra chilena. Universitas Psychologica, 9(1), 161-168.

Claggett, W. \& Pollock III, P. H. (2006). The modes of participation revisited, 1980-2004. Political Research Quarterly, 59(4), 593600. https://doi.org/10.1177/106591290605900408 
Cohen, A., Vigoda, E. \& Samorly, A. (2001). Analysis of the mediating effect of personal - psychological variables on the relationship between socio-economic status and political participation: A structural equations framework. Political Psychology, 22(4), 727- 757. https://doi.org/10.1111/0162-895X.00260

Crowson, H.M., Thoma, S.J. \& Hestevold, N. (2005). Is political conservatism synonymous with authoritarianism? The Journal of Social Psychology, 145(5), 571-592. https://doi.org/10.3200/ SOCP.145.5.571-592

Dalton, R. J. (2006). Citizenship norms and political participation in America: The good news is ... the bad news is wrong. CDACS Occasional Paper. Irvine: Center for the Study of Democracy, University of California.

Dalton, R.J. (2008). Citizenship norms and the expansion of political participation. Political Studies, 56, 76-98. https://doi. org/10.1111/j.1467-9248.2007.00718.x

Delfino, G., Zubieta, E.M. \& Muratori, M. (2013). Tipos de participación política: análisis factorial confirmatorio con estudiantes universitarios de Buenos Aires, Argentina. Psicología Política, 13(27), 301-318.

Delli Carpini, M. \& Keeter, S. (1993). Measuring political knowledge: putting first things first. American Journal of Political Science, 37(4), 1179/1206. https://doi.org/10.2307/2111549

Duckitt, J. \& Fisher, K. (2003). The impact of social threat on worldview and ideological attitudes. Political Psychology, 24(1), 199-222. https://doi.org/10.1111/0162-895X.00322

Duckitt, J., Wagner, C., du Plessis, I. \& Birum, I. (2002). The psychological bases of ideology and prejudice: Testing a dual process model. Journal of Personality and Social Psychology, 83(1), 75-93. https://doi.org/10.1037/0022-3514.83.1.75

Fernández-Prados, J.S. \& Rojas-Tejada, A. J. (2003). Analysis of the unconventional political action scale: Results in Spain. Field Methods, 15(2), 131-142. 
França, D.X. (1995). O comportamento político: um a análise psicosocial em termos de expectativas. Disertación para optar al grado de máster. João Pessoa: Universidade Federal da Paraíba.

Gabriel, O.W. \& van Deth, J.W. (1998). Political interest. En J. van Deth \& E. Scarbrough (Eds.), The Impact of Values (pp. 390-411). New York: Oxford University Press. https://doi. org/10.1093/0198294751.003.0014

Gallego, A. (2007). Inequality in political participation: Contemporary patterns in European countries. Paper 07-01. Irvine: Center for the Study of Democracy, University of California.

Grossi Queipo, F. J. \& Ovejero Bernal, A. (1994). Alienación y participación política en la universidad de Oviedo. Psicología Política, 8, 45-61.

Hair, J. F., Anderson, R.E., Tatham, R.L. \& Black, W.C. (1999). Análisis Multivariante (5ta. ed.). Madrid: Prentice Hall Iberia.

Heaven, P.C.L. \& Bucci, S. S. (2001). Right-wing authoritarianism, social dominance orientation and personality: an analysis using the IPIP measure. European Journal of Personality, 15(1), 49-56. https://doi.org/10.1002/per.389

Hui, C.H. (1988). Measurement of individualism-collectivism. Journal of Research in Personality, 22(1), 17-36. https://doi.org/ 10.1016/0092-6566(88)90022-0

John, P., Fieldhouse, E. \& Liu, H. (2011). How civic is the civic culture? Mapping the routes to community participation using 2005 English Citizenship Survey. Political Studies, 59(2), 230252. https://doi.org/10.1111/j.1467-9248.2011.00891.x

Jost, J.T. \& Hunyady, O. (2002). The psychology of system justification and the palliative function of ideology. European Review of Social Psychology, 13, 111-153. https://doi.org/ 10.1080/10463280240000046

Jost, J., Glaser, J., Kruglanski, A. \& Sulloway, F. (2003). Political conservatism as motivated social cognition. Psychological Bulletin, 129(3), 339-375. https://doi.org/10.1037/00332909.129.3.339 
Kish, L. (1965). Survey Sampling. New York: Wiley.

Klesner, J.L. (2007). Social capital and political participation in Latin America. Evidence from Argentina, Chile, Mexico and Peru. Latin American Research Review, 42(2), 1-32. https://doi. org/10.1353/lar.2007.0022

Komarraju, M. \& Cokley, K.O. (2008). Horizontal and vertical dimensions of individualism-collectivism: A comparison of African Americans and European Americans. Cultural Diversity and Ethnic Minority Psychology, 14(4), 336-343. https://doi. org/10.1037/1099-9809.14.4.336

Krampen, G. (2000). Transition of adolescent political action orientations to voting behavior in early adulthood in view of a social-cognitive action theory model of personality. Journal of Political Psychology, 21(2), 277-297. https://doi. org/10.1111/0162-895X.00188

Lehmiller, J.J. \& Schmitt, M.T. (2007). Group domination and inequality in context: Evidence for the unstable meanings of social dominance and authoritarianism. European Journal of Social Psychology, 37(4), 704-724. https://doi.org/10.1002/ejsp.383

Letki, N. (2002). Lustration and consolidation of democracy in EastCentral Europe. Europe-Asia Studies, 54(4), 529-52. https://doi. org/10.1080/09668130220139154

Mannarini, T., Legittimo, M. \& Talò, C. (2008). Determinants of social and political participation among youth. A preliminary study. Psicología Politica, 36, 95-117.

Marien, S., Hooghe, M. \& Quintelier, E. (2010). Inequalities in noninstitutionalized forms of political participation. A multilevel analysis for 25 countries. Political Studies, 58(1), 187-213. https://doi.org/10.1111/j.1467-9248.2009.00801.x

Mavor, K.I., Louis, W.R. \& Sibley, C. G. (2010). A bias-corrected exploratory and confirmatory factor analysis of right-wing authoritarianism: Support for a three-factor structure. Personality and Individual Differences, 48(1), 28-33. https://doi. org/10.1016/j.paid.2009.08.006 
McAtee, A. \& Wolak, J. (2011). Why people decide to participate in state politics. Political Research Quarterly, 64(1), 45-58. https:// doi.org/10.1177/1065912909343581

Milner, H. (2010). The internet generation: engaged citizens or political dropouts. Líbano: Tufts University Press.

Morales, L. (2005). ¿Existe una crisis participativa? La evolución de la participación política y el asociacionismo en España. Revista Española de Ciencia Política, 13, 51-87.

Morales, L. (2009). Joining political organizations. Institutions, mobilization and participation in western democracies. Colchester: ECPR Press.

Morrell, M.E. (2003). Survey and experimental evidence for a reliable and valid measure of internal political efficacy. Public Opinion Quarterly, 67(4), 589-602. https://doi.org/10.1086/378965

Norris, P. (2002). Democratic Phoenix: Reinventing political activism. New York: Cambridge University Press. https://doi.org/10.1017/ CBO9780511610073

Oyserman, D. (1993). The lens of personhood: Viewing the self, others, and conflict in a multicultural society. Journal of Personality and Social Psychology, 65, 993-1009. https://doi.org/10.1037/00223514.65.5.993

Paquet, S. L. \& Kline, T. J. B. (2009). Uncovering the psychometric properties of scales measuring individualist and collectivist orientations. International Journal of Testing, 9(3), 260-270. https://doi.org/10.1080/15305050903106859

Pearl, D.S. \& Anderson, R. K. (2007). Social isolation and student voting behavior. How voting frequency is affected by individual perceptions of loneliness. Psicología Politica, 35, 87-100.

Reef, M.J. \& Knoke, D. (1999). Political alienation and efficacy. En J. P. Robinson, P. R. Shaver y L. S. Wrightsman (Eds.), Measures of Political Attitudes (pp. 413-464). Londres: Academic Press.

Robnett, B. (2007). Does collective identity matter? African-American conventional and unconventional political participation, Paper 
07-05. Irvine: Center for the Study of Democracy, University of California.

Rodríguez, M., Sabucedo, J.M. \& Costa, M. (1993). Factores motivacionales y psicosociales asociados a distintos tipos de acción política. Psicología Política, 7, 19-38.

Sabucedo, J. M. \& Arce, C. (1991). Types of political participation: A multidimensional analysis. European Journal of Political Research, 20(1), 93-102. https://doi.org/10.1111/j.1475-6765.1991. tb00257.x

Sabucedo, J. M. (1996). Psicología Politica. Madrid: Síntesis.

Schimmack, U., Oishi, S. \& Diener, E. (2005). Individualism: A valid and important dimension of cultural differences between nations. Personality and Social Psychology Review, 9(1), 17-31. https://doi.org/10.1207/s15327957pspr0901_2

Schwartz, S.H. (2006). A theory of cultural value orientations: Explication and applications. Comparative Sociology, 5(2-3), 137-182. https://doi.org/10.1163/156913306778667357

Shulruf, B., Hattie, J. \& Dixon, R. (2007). Development of a new measurement tool for individualism and collectivism. Journal of Psychoeducational Assessment, 25(4), 385-401. https://doi. org/10.1177/0734282906298992

Sibley, C. G. \& Duckitt, J. (2009). Big-five personality, social worldviews and ideological attitudes: Further tests of a dual process cognitivemotivational model. The Journal of Social Psychology, 149(5), 545-561. https://doi.org/10.1080/00224540903232308

Somuano Ventura, M. F. (2005). Más allá del voto: modos de participación política no electoral en México. Foro Internacional, XLV(1), 65-88.

Sorribas, P.M. (2012) Participación Politica: desarrollo de un modelo explicativo desde el enfoque de la cognición social. Disertación para optar al grado de Doctor, Universidad Nacional de Córdoba.

Sorribas, P.M. (2014). La participación política. Dimensionalidad, amplitud, nivel de involucramiento y factores predictivos. Aportes desde la Psicología Política al desarrollo de la demo- 
cracia participativa. En S. R. Ilari, P. Sorribas y Y. Guthmann, III Premio a la innovación y mejoramiento de las politicas públicas: Categoría doctorado (pp. 45-97). Ciudad Autónoma de Buenos Aires: Instituto Nacional de la Administración Pública - INAP.

Soule, S. (2001). Will they engage? Political knowledge, participation and attitudes of Generations X and Y. Trabajo presentado en el Active Participation or a Retreat to Privacy, Potsdam, Germany. Teorell, J. (2003). Linking social capital to political participation: Voluntary associations and networks of recruitment in Sweden. Scandinavian Political Studies, 26(1), 49-66. https://doi. org/10.1111/1467-9477.00079

Torcal, M., Montero, J. R. \& Teorell, J. (2006). La participación política en Espańa: modos y niveles en perspectiva comparada. En J. R. Montero, J. Font \& M. Torcal (Eds.), Ciudadanos, asociaciones y participación en España (pp. 47-75). Madrid: CIS.

Van Der Meer, T. \& Van Ingen, E. (2009). Schools of democracy? Disentangling the relationship between civic participation and political action in 17 European countries. European Journal of Political Research, 48, 281-308. https://doi.org/10.1111/j.14756765.2008.00836.x

Van der Meer, T., van Deth, J. W. \& Scheepers, P. L. H. (2009). The politicized participant: Ideology and political action in 20 democracies. Comparative Political Studies, 42(11), 1426-1457. https://doi.org/10.1177/0010414009332136

Van Hiel, A., Cornelis, I., Roets, A. \& De Clercq, B. (2007). A comparison of various authoritarianism scales in Belgian Flanders. European Journal of Personality, 21(2), 149-168. https://doi.org/10.1002/per.617

Velásquez, E. \& Martínez, L. (2004). Participación y género: desafíos para el desarrollo de una política de juventud en Chile. Psykhe, 13(1), 43-51. https://doi.org/10.4067/S071822282004000100004

Velásquez, E., Martínez, L. M. \& Cumsille, P. (2004). Expectativas de autoeficacia y actitud prosocial asociadas a participación ciuda- 
dana en jóvenes. Psykhe, 13(2), 85-98. https://doi.org/10.4067/ S0718-22282004000200007

Verba, S. \& Nie, N. (1972). Participation in America: Political democracy and social equality. New York: Harper \& Row.

Vráblíková, K. (2010). Contextual determinants of political participation in democratic countries. Trabajo presentado en Conference on Civic, Political and Cultural Engagement Among Migrants, Minorities and National Populations: Multidisciplinary Perspectives, Centre for Research on Nationalism, Ethnicity and Multiculturalism, University of Surrey, junio, Guildford, UK.

Walter, M. I., Stone, W.F. \& Bourgeois, D. Y. (1996). Autoritarismo y estilo de respuesta. Nuevos resultados sobre una vieja cuestión. Psicología Politica, 13, 17-27.

Weber, C. \& Federico, C. M. (2007). Interpersonal attachment and patterns of ideological belief. Political Psychology, 28(4), 389416. https://doi.org/10.1111/j.1467-9221.2007.00579.x

Whiteley, P. \& Seyd, P. (2002). High intensity participation - The dynamics of party activism in Britain. Ann Arbor, MI: University of Michigan Press. https://doi.org/10.3998/mpub.14704

Wollman, N. \& Stouder, R. (1991). Believed efficacy and political activity: A test of the specificity hypothesis. Journal of Social Psychology, 131(4), 557-567. https://doi.org/10.1080/0022454 5.1991 .9713885

Zmerli, S. (2002). Bonding and bridging social capital. A relevant concept for political participation? Trabajo presentado en la ECPR Joint Sessions, Workshop N ${ }^{\circ}$. 7, Turin, Italy.

Recibido: 05 de junio del 2016 Revisado: 30 de septiembre, 2016 Aceptado: 17 de octubre, 2016 